\title{
Adrenal tumor volume in a genetically engineered mouse model of neuroblastoma determined by magnetic resonance imaging
}

\author{
KUMI KAWANO $^{1}$, YOSHIYUKI HATTORI ${ }^{1}$, HIROSHI IWAKURA ${ }^{2}$, TAKASHI AKAMIZU ${ }^{3}$ and YOSHIE MAITANI $^{1}$ \\ ${ }^{1}$ Institute of Medicinal Chemistry, Hoshi University, Shinagawa-ku, Tokyo 142-8501; \\ ${ }^{2}$ Medical Innovation Center, Kyoto University Graduate School of Medicine, Yoshida-Konoe-Cho, Sakyo-ku, Kyoto 606-8501; \\ ${ }^{3}$ The First Department of Medicine, Wakayama Medical University, Wakayama 641-8509, Japan
}

Received March 8, 2012; Accepted April 24, 2012

DOI: $10.3892 /$ etm.2012.564

\begin{abstract}
Neuroblastoma is the second most common type of solid tumor in children and is commonly found in the adrenal medulla. Recently, we developed transgenic mice exhibiting tumors bilaterally in the adrenal medulla through the expression of SV40 T-antigen. Since these transgenic mice facilitate the development of new therapeutic approaches for neuroblastoma, non-invasive monitoring methods are required for serial measurement of tumor progression. In this study, we monitored the serial progression of adrenal tumors in transgenic mice by magnetic resonance imaging (MRI) of $9.4 \mathrm{~T}$ vertical type, and calculated the tumor volume. The accuracy of the tumor volume determination by MRI was verified by standard volume measurements at autopsy. Adrenal tumors as small as $1.5 \mathrm{~mm}$ in diameter were detected and quantitatively measured in the transgenic mice by in vivo MRI without using exogenous contrast agents on $\mathrm{T}_{2}$-weighted spin echo images. The tumor sizes by MRI correlated better with tumor weight than the volume by calculation with a caliper. Furthermore, we monitored the change of tumor volume following administration of doxorubicin at weekly intervals. The tumor progression and regression following doxorubicin treatment in the individual mice could be observed by serial MRI. From these findings, non-invasive MRI is likely to be useful for monitoring the response of spontaneous tumors to therapeutic drugs.
\end{abstract}

\section{Introduction}

Several tumor models created by subcutaneous, intravenous, or orthotopic injection of human tumor cells into immunodeficient mice have been developed for the evaluation of the therapeutic effects of chemotherapy (1-3). Preclinical studies

Correspondence to: Dr Kumi Kawano, Institute of Medicinal Chemistry, Hoshi University, Ebara 2-4-41, Shinagawa-ku, Tokyo 142-8501, Japan

E-mail:kkumi@hoshi.ac.jp

Key words: adrenal gland, tumor size, magnetic resonance imaging, neuroblastoma, transgenic mouse model of therapeutic drugs for tumors have traditionally relied on xenografts of human tumor cells in severe combined immunodeficient (SCID) or athymic nude mice $(4,5)$. However, a tumor is composed not only of transformed cells, but is also intimately associated with host cells such as endothelial cells, fibroblasts and inflammatory cells that may potentially influence tumor growth. The tumor microenvironment is related to clinical prognoses, metastatic potential, and treatment-related outcomes. Subcutaneous implantation of human tumor cells allows quantitative measurements of tumor growth; however, the characteristics of the implanted tumors are different from those of spontaneous tumors since the tumors are not grown in physiological conditions due to the lack of a typical tumor microenvironment and immune system. As human tumor cells must be grown in immunocompromised recipients, the important effects of native immunity on disease initiation and progression cannot be studied in the xenograft model.

Recent advances in technology for the development of genetically engineered transgenic mice have resulted in the development of a number of cancer models. These models maintain an intact immune system. A disadvantage for the use of transgenic mice is the difficulty in the direct visualization of primary tumor growth when tumors are small and located at a site deep inside the body. Without reliable methods for tumor detection in transgenic mice, a large number of animals subjected to euthanasia and autopsy at the endpoint are required for evaluation of antitumor activity. Therefore, a non-invasive method for detecting tumors and measuring tumor volume is required. Magnetic resonance imaging (MRI) has been proposed as a method to visualize and monitor tumor development. The advantages of MRI are that it provides a noninvasive method for tumor detection, high-resolution images of anatomical structures, and the means for accurate serial measurement of solid tumor volumes in animal models $(3,6)$.

Neuroblastoma is the second most common type of solid tumor in children (7). Most children with lower-stage disease have a favorable outcome through surgery alone or following treatment with chemotherapy. However, tumors have a poor prognosis when they are unresectable or metastatic and diagnosed after one year of age (8). Therefore, the availability of reliable tumor models for adrenal neuroblastoma to test novel chemotherapeutic agents remains crucial for improving survival. Recently, we developed transgenic mice exhibiting 
tumors in bilateral adrenal glands with histological characteristics similar to human neuroblastoma (9). Comparison of the gene expression profiles by DNA microarray also indicated that the character of the mouse adrenal tumors was similar to that of human adrenal neuroblastoma rather than pheochromacytoma (10). In this study, we monitored the development of adrenal tumors in individual mice by MRI, and the accuracy of MRI-based tumor volume determinations was verified by standard volume measurements at autopsy. Furthermore, the change of tumor volume following doxorubicin treatment was evaluated by MRI analysis. This information aids evaluation of the efficacy of new treatments for neuroblastoma.

\section{Materials and methods}

Transgenic mice. Transgenic mice exhibiting spontaneous bilateral adrenal tumors with a C57BL/6 background were previously developed (9). The transgenic mice carried tetracycline-inducible simian virus $40 \mathrm{~T}$-antigen, a fusion gene comprising tetracycline-responsive elements with cytomegalovirus promoter and simian virus $40 \mathrm{~T}$-antigen. Transgenic mice were used as heterozygotes. All experimental procedures were approved by the Committee on Animal Research of Hoshi University.

Magnetic resonance imaging. Mice were anesthetized with $1.5 \%$ isoflurane (Abbott Japan, Tokyo, Japan) throughout the MRI experiment during their insertion into a $9.4 \mathrm{~T}$ vertical-type MRI (Varian MRI System, Varian, Palo Alto, CA, USA). Two-dimensional $\mathrm{T}_{2}$-weighted images were obtained using a multi-slice spin echo sequence (repetition time/echo time, $1000 \mathrm{msec} / 40 \mathrm{msec}$ ) with a field of view of $30 \times 30 \mathrm{~mm}$, a matrix of 256x256 pixels, and a slice thickness of $1 \mathrm{~mm}$.

In order to minimize the imaging time of a large number of mice, a fast spin echo sequence was used for the tumor response following doxorubicin treatments. Coronal $\mathrm{T}_{2}$-weighted images were obtained using multi-slice fast spin echo sequence (repetition time/effective echo time, $2500 \mathrm{msec} / 48 \mathrm{msec}$ ) with a field of view of $50 \times 30 \mathrm{~mm}$, a matrix of $256 \times 256$ pixels, and a slice thickness of $1 \mathrm{~mm}$.

Tumor volume measurement. ImageJ software (NIH, Bethesda, MD, USA) was used for image processing and analysis. To determine the volume of the adrenal tumors, each image was reviewed and regions of interest (ROIs) were identified as adrenal glands. The ROIs corresponding to the right and left adrenal glands were defined individually, and the volume of each adrenal gland was calculated by summing up the ROIs in consecutive slices. Following MRI scan, tumor weights were recorded and the length of the tumor was measured with a caliper. In this measurement with a caliper, tumor volume was calculated using the following formula: $\mathrm{LxW}^{2} \mathrm{x} \pi / 6$, where $\mathrm{L}$ is the long diameter and $\mathrm{W}$ is the short diameter of the tumor.

Chemotherapy with doxorubicin. To evaluate the therapeutic response to doxorubicin treatment, mice were subjected to weekly MRI scans starting at 13 weeks of age. Transgenic mice were randomly assigned to receive three intravenous injections of doxorubicin $(3 \mathrm{mg} / \mathrm{kg})$ at weekly intervals or intravenous injection of saline as a control.

\section{Results}

Magnetic resonance imaging. In our previous study we reported on the ectopic expression of SV40 T-antigen in adrenal medulla which developed bilateral large adrenal tumors in 12-13 week-old mice (9). Transgenic mice, beginning at 13 weeks of age, developed carcinoma of the adrenal gland (Fig. 1A), and by 15 weeks of age, most adrenal tumors were between 5 and $10 \mathrm{~mm}$ in diameter. At 17 weeks of age, tumors of the adrenal glands had enlarged to $10-15 \mathrm{~mm}$ in diameter. Next, we evaluated the adrenal tumor progression of transgenic mice by MRI as a non-invasive monitoring method. The tumor was hyperintense on $\mathrm{T}_{2}$-weighted images relative to $\mathrm{T}_{1}$-weighted ones (data not shown); therefore, we followed the growth of adrenal tumors by coronal $\mathrm{T}_{2}$-weighted images without exogenous contrast agents (Fig. 1B). The adrenal glands of normal mice were barely evident in this procedure due to their small size. In contrast, the adrenal tumors in the transgenic mice after 13 weeks of age were detected above the kidneys and were easily delineated from adjacent organs.

To assess the accuracy of the adrenal tumor volumetric determination by MRI, we calculated the tumor volumes from MRI data and compared these with tumor wet weights and tumor volumes calculated by caliper measurement (Fig. 2). Linear regression analyses demonstrated that tumor volume determined by MRI correlated well with tumor wet weight $(r=0.996)$ and caliper measurement $(r=0.944)$. These findings indicate that tumor volume in transgenic mice can be quantitatively measured by MRI as well as by weighing.

Adrenal tumors in transgenic mice at 13 weeks of age were detected by MRI (Fig. 1). Therefore, we assessed the growth of individual tumors by sequential MRI in mice from 12 to 15 weeks of age (Fig. 3). The sizes of all the tumors in the transgenic mice were detectable at 12 weeks of age, and increased over time. Although the total tumor growth curves for each of the three groups of mice were similar, there was considerable variation in the volume of individual tumors for each mouse. In subsequent evaluation of tumor growth by treatment with antitumor drug, the sum of right and left adrenal tumor volumes was used to represent the tumor volume of each mouse.

Tumor progression and regression by doxorubicin treatment. Finally, we examined the effects of a therapeutic drug on transgenic mice with the primary objective of determining whether adrenal tumor progression would be delayed or if tumors would regress in treated mice compared with those of the controls (saline injection). In a previous study, we found that the expression of DNA topoisomerase II $\alpha$ (Topo II $\alpha$ ) mRNA in the adrenal tumors of transgenic mice was strongly increased compared with that in non-transgenic mice (10); therefore, we evaluated the therapeutic effect of doxorubicin, which is an inhibitor of Topo II $\alpha$ (Fig. 4). MRI with a fast spin echo sequence minimized the scan time, without loss of image resolution necessary for size determination. As a result, tumor volume regression was observed in mice treated with doxorubicin and the size of a number of tumors was under the detection limit $(\sim 1.5 \mathrm{~mm})$. In tumor sections following injection of doxorubicin, large areas of necrosis were observed (data not shown). These findings corresponded with the prognostic results from the DNA array. However, tumor regrowth was observed at approximately 
Normal

A

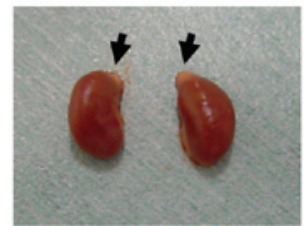

$\mathbf{B}$

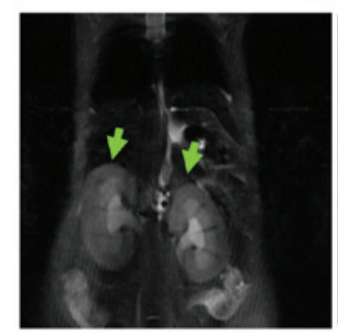

$13 \mathrm{~T}$
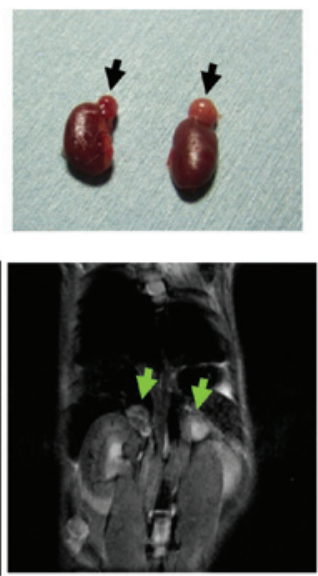

$15 \mathrm{~T}$
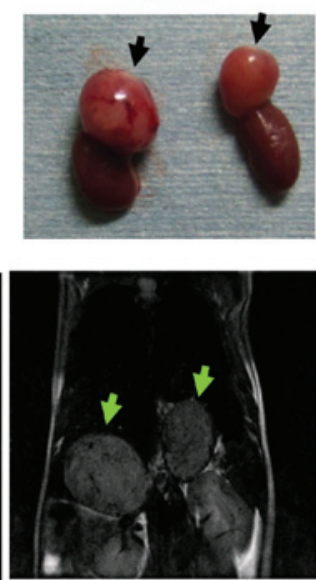

$17 \mathrm{~T}$
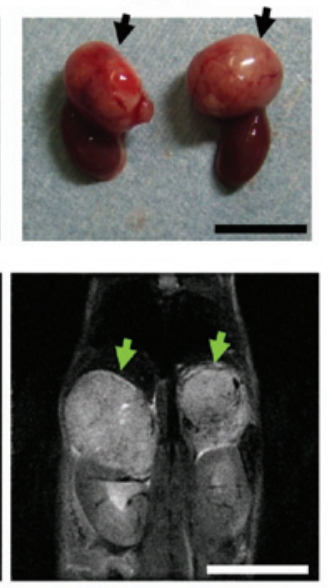

Figure 1. Images of adrenal tumors in a transgenic mouse by MRI. (A) Gross appearances and (B) $\mathrm{T}_{2}$-weighted coronal images from a transgenic mouse at the ages of 13,15 , and 17 weeks (13T, 15T, and 17T, respectively) and normal adrenal glands from a non-transgenic littermate at the age of 13 weeks. Arrows indicate the adrenal glands in the non-transgenic littermate and adrenal tumors in a transgenic mouse. Bars, $10 \mathrm{~mm}$.

A

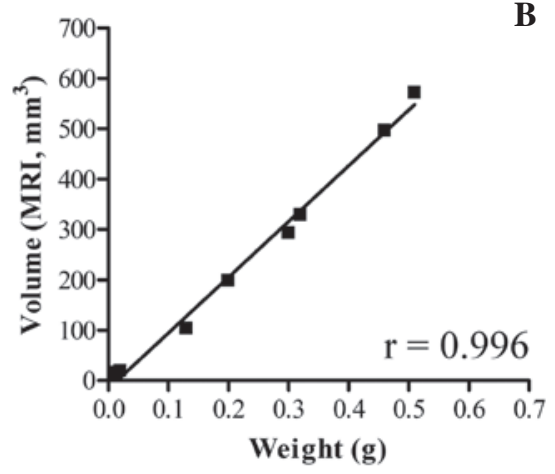

B

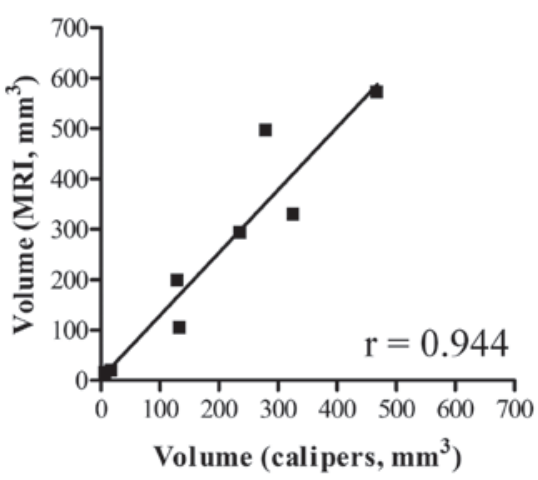

Figure 2. Tumor volumes from MRI data in comparison to tumor wet weights (A) and tumor volumes calculated by caliper measurement (B). $\mathrm{n}=8$.

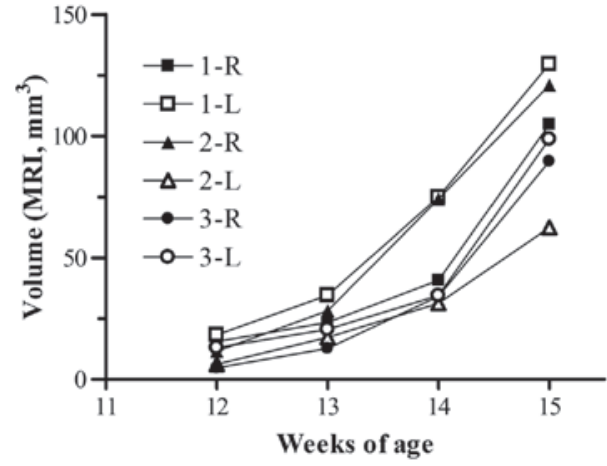

Figure 3. Tumor volumetric analysis in transgenic mice by serial MRI. The volumes of right $(\mathrm{R})$ and left $(\mathrm{L})$ adrenal tumors were determined by weekly imaging for three individual mice (1-3) from 12 to 15 weeks of age.

19 weeks of age. We could evaluate the continuous change in tumor size following drug treatment using MRI.

\section{Discussion}

Transgenic mice exhibiting spontaneous tumors are a useful model for evaluation of the efficacy of anticancer drug

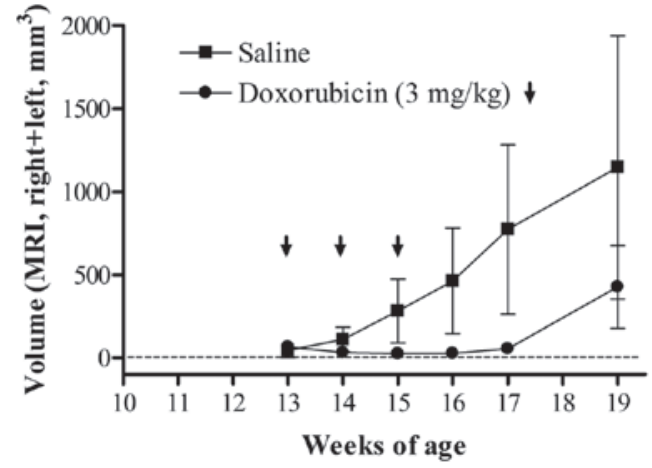

Figure 4. Adrenal tumor volume after doxorubicin treatment. Transgenic mice were administered $3 \mathrm{mg} / \mathrm{kg}$ doxorubicin (closed circle) or saline (closed square) at weekly intervals three times (at 13, 14, and 15 weeks of age). The volumes were calculated as the sum of right and left adrenal tumors. Each value represents the mean of six mice and the standard deviation $(n=3)$.

treatment. Since spontaneous tumors are embedded in a microenvironment that closely mimics that of human disease, reliable methods for measurement of tumor size are required. Tumor volume determination by autopsy is not suited for sequential observation. MRI permits a wide variety of longitudinal 
studies not possible with other destructive analytical methods. Recently, MRI for tumors has become more readily available in cancer research and has been used for several transgenic models including lung (11) and ovarian tumors (6). Transgenic tumor models have been evaluated by MRI not only to assess tumor size but also to evaluate therapeutic response $(6,12)$. However, to the best of our knowledge, there is little information regarding quantitative measurement of tumor volume by MRI. In this study, we monitored the adrenal tumor progression of transgenic mice by MRI. To establish a non-invasive MRI scanning protocol that has high resolution for tumors, we characterized the intrinsic MR contrast parameter $\left(\mathrm{T}_{2}\right)$ of the adrenal tumors. Adrenal tumors were easily identifiable without using exogenous contrast agents on $\mathrm{T}_{2}$-weighted spin echo images. We established a correlation between tumors detected by MRI and those observed ex vivo following harvesting of the adrenal tumors. Tumor volume determined by MRI was better correlated with that by weighing $(r=0.996)$ than that by caliper measurements $(r=0.944)$, indicating that MRI is able to measure the tumor volume three-dimensionally and, therefore, more accurately than two-dimensional measurement. Although we used MRI with a high resolution with a $9.4 \mathrm{~T}$ instrument, there was limited detection of small tumors $(1.5 \mathrm{~mm}$ in diameter and less than $2 \mathrm{~mm}^{3}$ in volume). An MR instrument with a stronger magnetic field or the use of exogenous contrast agents might provide higher resolution of much smaller tumors in a small animal model (3).

Researchers are studying chemotherapy drugs in order to find an effective therapy for neuroblastoma. In chemotherapy for high-risk neuroblastoma, the following drugs are often used: Cyclophosphamide, ifosfamide, cisplatin, carboplatin, vincristine, doxorubicin, melphalan, etoposide, teniposide, and topotecan. In previous study, we found that the expression of DNA Topo II $\alpha$ mRA in adrenal tumors of transgenic mice increased 120- to 150-fold compared with that in non-transgenic mice (10); therefore, we evaluated the therapeutic effect of doxorubicin. As expected from the gene expression profile (10), delay of adrenal tumor progression by doxorubicin treatment was observed by serial MRI. Furthermore, therapeutic response, including tumor regression and regrowth, was also measured using longitudinal MRI. MRI provides important insights into the factors that control the onset and development of tumors and serves as an important platform for the preclinical development and evaluation of novel chemotherapeutic agents having a high likelihood of efficacy in human clinical trials.

In conclusion, MRI is a powerful imaging modality for the in vivo characterization of adrenal tumors in mice. The use of repeated scans by MRI in the same animal reduces sample size, and this method enables efficient screening of novel therapeutic approaches for neuroblastoma.

\section{Acknowledgements}

We thank Ms. Yuko Iwase, Ms. Kimiko Koga, Mr. Yuki Shimojo, Ms. Yukimi Taniguchi, Mr. Takuya Minowa, Mr. Tomohiro Izumisawa, and Mr. Haruya Ubukata for their assistance with the experimental work. This research was supported in part by grants from the Ministry of Education, Culture, Sports, Science and Technology, Japan, the Ministry of Health, Labour and Welfare, Japan, the Science Research Promotion Fund from the Promotion and Mutual Aid Corporation for Private Schools of Japan, and the Open Research Center Project.

\section{References}

1. Talmadge JE, Singh RK, Fidler IJ and Raz A: Murine models to evaluate novel and conventional therapeutic strategies for cancer. Am J Pathol 170: 793-804, 2007.

2. Zhang L, Smith KM, Chong AL, Stempak D, Yeger H, Marrano P, Thorner PS, Irwin MS, Kaplan DR and Baruchel S: In vivo antitumor and antimetastatic activity of sunitinib in preclinical neuroblastoma mouse model. Neoplasia 11: 426-435, 2009.

3. Moats R, Ma LQ, Wajed R, Sugiura Y, Lazaryev A, Tyszka M, Jacobs R, Fraser S, Nelson MD Jr and DeClerck YA: Magnetic resonance imaging for the evaluation of a novel metastatic orthotopic model of human neuroblastoma in immunodeficient mice. Clin Exp Metastasis 18: 455-461, 2000.

4. Connolly DC, Bao R, Nikitin AY, Stephens KC, Poole TW, Hua X, Harris SS, Vanderhyden BC and Hamilton TC: Female mice chimeric for expression of the simian virus 40 TAg under control of the MISIIR promoter develop epithelial ovarian cancer. Cancer Res 63: 1389-1397, 2003.

5. Bock NA, Zadeh G, Davidson LM, Qian B, Sled JG, Guha A and Henkelman RM: High-resolution longitudinal screening with magnetic resonance imaging in a murine brain cancer model. Neoplasia 5: 546-554, 2003.

6. Hensley H, Quinn BA, Wolf RL, Litwin SL, Mabuchi S, Williams SJ, Williams C, Hamilton TC and Connolly DC: Magnetic resonance imaging for detection and determination of tumor volume in a genetically engineered mouse model of ovarian cancer. Cancer Biol Ther 6: 1717-1725, 2007.

7. Maris JM,Hogarty MD, Bagatell R and Cohn SL: Neuroblastoma. Lancet 369: 2106-2120, 2007.

8. Brodeur GM: Neuroblastoma: biological insights into a clinical enigma. Nat Rev Cancer 3: 203-216, 2003.

9. Iwakura H, Ariyasu H, Kanamoto N, Hosoda K, Nakao K, Kangawa K and Akamizu T: Establishment of a novel neuroblastoma mouse model. Int J Oncol 33: 1195-1199, 2008.

10. Hattori Y, Kanamoto N, Kawano K, Iwakura H, Sone M, Miura M, Yasoda A, Tamura N, Arai H, Akamizu T, Nakao K and Maitani Y: Molecular characterization of tumors from a transgenic mouse adrenal tumor model: Comparison with human pheochromocytoma. Int J Oncol 37: 695-705, 2010.

11. Degrassi A, Russo M, Nanni C, Patton V, Alzani R, Giusti AM, Fanti S, Ciomei M, Pesenti E and Texido G: Efficacy of PHA-848125, a cyclin-dependent kinase inhibitor, on the K-Ras(G12D)LA2 lung adenocarcinoma transgenic mouse model: evaluation by multimodality imaging. Mol Cancer Ther 9: 673-681, 2010.

12. He Z, Evelhoch JL, Mohammad RM, Adsay NV, Pettit GR, Vaitkevicius VK and Sarkar FH: Magnetic resonance imaging to measure therapeutic response using an orthotopic model of human pancreatic cancer. Pancreas 21: 69-76, 2000. 